Reprod. Nutr. Dévelop., 1988, 28 (1), 143-144.

\title{
Evolution de quelques paramètres digestifs et métaboliques chez le veau avant et après sevrage en relation avec la dégradabilité des protéines alimentaires
}

\author{
J. P. LALLES, C. PONCET
}

Laboratoire de la Digestion des Ruminants, I.N.R.A., Theix, 63122 Ceyrat, France.

Summary. Changes in ruminal fermentation patterns and of some blood parameters were studied in calves receiving concentrates with high or low degradability nitrogen, between 7 and 20 weeks (weaning at 10 weeks). The groups differed only for ruminal $\mathrm{N}-\mathrm{NH}_{3}$ and blood urea concentrations.

L'évolution de la digestion dans le rumen du veau autour du sevrage a été suivie à travers l'évolution des principaux paramètres fermentaires et métaboliques entre la $5^{e}$ et la $20^{e}$ semaine, sur des animaux sevrés à 10 semaines. On a comparé deux sources de protéines de l'aliment concentré de dégradabilité dans le rumen très élevée (pois) ou plus limitée (tourteau de soja).

Matériel et méthodes. Douze veaux mâles frisons fistulés à l'âge de 3 semaines ont ingéré $900 \mathrm{~g}$ de matière sèche (MS) d'un aliment d'allaitement jusqu'à l'âge de 6 semaines puis $450 \mathrm{~g}$ jusqu'à 10 semaines. A partir de la 7e semaine, ils ont reçu du foin de graminées (F) (2 fois par jour) et un aliment concentré $(C)$ ( 8 fois par jour à intervalle de $3 \mathrm{~h}$ ) constitué de pois (lot $P$ ) ou de tourteau de soja (lot S) mélangé avec de l'amidon de maïs pour obtenir des teneurs identiques en énergie (2,8 Mcal d'énergie métabolisable) et en matières azotées $(18 \%)$ dans la MS. Les proportions de $F$ et $C(20: 80)$ ont été maintenues entre les périodes de mesures en rationnant le foin et pendant les périodes de mesures en limitant l'ingestion au niveau observé pendant les 4 jours précédant ces périodes. Les deux sources de protéines comparées représentaient $92 \%$ de l'azote des aliments secs ingéré. Les prélèvements de liquide du rumen et de sang jugulaire ont été effectués aux semaines 5-6 $\left(P_{1}\right), 9-10\left(P_{2}\right), 15-16\left(P_{3}\right)$, 19-20 $\left(\mathrm{P}_{4}\right)$ à $15 \mathrm{~h}, 1 \mathrm{~h} 30$ après la distribution de concentré. Les analyses effectuées sont indiquées dans le tableau 1. Les différences entre lots et entre périodes ont été analysées respectivement par les tests $U$ de Mann et Whitney et $\mathrm{T}$ de Wilcoxon.

Résultats et discussion. Au cours de la phase lactée, l'accroissement de la consommation d'aliment sec se traduit par une augmentation importante de la teneur ruminale en acides gras volatils, une baisse de la teneur en $\mathrm{N}_{-} \mathrm{NH}_{3}$ et du $\mathrm{pH}$. Ces trois paramètres n'évoluent plus après le sevrage. Au niveau sanguin, seules les teneurs en acétate $\left(C_{2}\right)$ et $\beta$-hydroxybutyrate $(\beta \mathrm{OH})$ augmentent régulièrement de $P_{1}$ à $P_{4}\left(C_{2}\right)$ et de $P_{1}$ à $P_{3}(\beta O H)$, témoignant du développement fonctionnel et métabolique progressifs du rumen pendant et après sevrage. La glycémie chute au moment du sevrage puis se stabilise alors que l'insulinémie diminue transitoirement pendant cette période. L'urémie baisse pendant la phase lactée, augmente ensuite $\left(P_{3}\right)$ et se stabilise (lot $P$ ) ou diminue (lot $S$ ) en $P_{4}$.

Le lot $S$ se distingue du lot $P$ par des teneurs en $N-N_{3}$ ruminal de $P_{2}$ à $P_{4}$ systématiquement plus faibles résultant certainement de la différence de dégradabilité des protéines des aliments concentrés. Ces faibles teneurs en $\mathrm{N}-\mathrm{NH}_{3}$ 
TABL. 1. - Evolution des quantités ingérées (MS et N), et des paramètres ruminaux et sanguins chez le veau sevré à 10 semaines, entre les âges de 5 et 20 semaines (moyennes (ETM)).

\begin{tabular}{|c|c|c|c|c|c|c|c|}
\hline $\begin{array}{l}\text { Régimes } \\
\text { (nb veaux) }\end{array}$ & Lait (12) & & Pois (6) & & & Soja (6) & \\
\hline Age (semaines) & $5-6$ & $9-10$ & $15-16$ & $19-20$ & $9-10$ & $15-16$ & $19-20$ \\
\hline $\begin{array}{l}\text { MS ingérée }(\mathrm{kg} / \mathrm{j}) \\
N \text { ingéré }(\mathrm{g} / \mathrm{j})\end{array}$ & $\begin{array}{c}0,9(0,1) \\
33 \quad\{1\}\end{array}$ & $\begin{array}{r}1,2(0,1) \\
35 \quad(2)\end{array}$ & $\begin{array}{r}1,5(0,1) \\
36 \quad(3)\end{array}$ & $\begin{array}{cc}2,3 & (0,2) \\
60 & (4)\end{array}$ & $\begin{aligned} 1,2 & (0,1) \\
36 & (2)\end{aligned}$ & $\begin{array}{rr}1,4 & (0,2) \\
35 & (2)\end{array}$ & $\begin{array}{r}2,2(0,3) \\
57 \quad(7)\end{array}$ \\
\hline $\begin{array}{l}\text { Rumen : } \\
\mathrm{N}-\mathrm{NH}_{3}(\mathrm{mg} / \mathrm{I}) \\
\mathrm{AGV} \text { totaux }(\mathrm{mM} / \mathrm{I}) \\
\mathrm{C}_{2}(\% \text { molaire }) \\
\mathrm{C}_{3}(\% \text { molaire }) \\
\mathrm{C}_{4}(\% \text { molaire }) \\
\mathrm{pH}\end{array}$ & $\begin{array}{cc}218 & (85) \\
15 & (5) \\
67 & (7) \\
22 & (5) \\
4 & (2) \\
7,1 & (0,1)\end{array}$ & $\begin{array}{rr}125 & (27) \\
119 & (17) \\
55 & (3) \\
32 & (3) \\
9 & (1) \\
6,0 & (0,2)\end{array}$ & $\begin{array}{rr}138 & (21) \\
114 & (12) \\
52 & (5) \\
29 & (7) \\
11 & (5) \\
6,2 & (0,3)\end{array}$ & $\begin{array}{rr}140 & (30) \\
127 & (25) \\
53 & (2) \\
28 & (3) \\
14 & (5) \\
6,0 & (0,3)\end{array}$ & $\begin{array}{rr}58 & (28) \\
116 & (13) \\
57 & (5) \\
28 & (6) \\
10 & (3) \\
6,0 & (0,4)\end{array}$ & $\begin{array}{rr}33 & (15) \\
124 & (15\rangle \\
57 & (6) \\
24 & (7) \\
12 & (2) \\
5,7 & (0,4)\end{array}$ & $\begin{array}{rr}55 & (22) \\
113 & (19) \\
58 & (7) \\
27 & (9) \\
11 & (3) \\
6,1 & (0,3)\end{array}$ \\
\hline $\begin{array}{l}\text { Sang : } \\
\text { Urée }(\mathrm{dg} / 1) \\
\mathrm{C}_{2}\left(10^{-5} \mathrm{M} / 1\right) \\
\beta \mathrm{OH}\left(10^{-5} \mathrm{M} / 1\right) \\
\text { Glucose }(\mathrm{dg} / 1) \\
\text { Insuline }(\mu \mathrm{M} / \mathrm{l})\end{array}$ & $\begin{array}{rr}2,1 & (0,3) \\
16 & (3) \\
5 & (5) \\
9,4 & (0,5) \\
27 & (10)\end{array}$ & $\begin{array}{rr}1,7 & (0,3) \\
26 & (10) \\
17 \quad(3) \\
7,9(0,6) \\
16 \quad(10)\end{array}$ & $\begin{array}{rr}2,4 & (0,6) \\
71 & (15) \\
43 & (6) \\
7,4 & (0,7) \\
15 & (3)\end{array}$ & $\begin{array}{rr}2,0 & (0,5) \\
107 & (17) \\
49 & (16) \\
7,7 & (1,1) \\
30 & (22)\end{array}$ & $\begin{array}{rr}1,7 & (0,5) \\
33 & (13) \\
16 & (5) \\
8,5 & (1,5) \\
18 & (8)\end{array}$ & $\begin{array}{rr}2,1 & (0,8) \\
63 & (19) \\
46 \quad(7) \\
7,3(1,0) \\
19 \quad(5)\end{array}$ & $\begin{array}{rr}1,2 & (0,3) \\
101 & (22) \\
46 & (16) \\
7,4 & (1,1) \\
22 & (8)\end{array}$ \\
\hline
\end{tabular}

sont susceptibles d'être limitantes pour la synthèse microbienne, comme l'ont observé Williams et Smith (1976) chez des veaux sevrés ingérant des aliments concentrés de teneur et dégradabilité en azote variables. La baisse de l'urémie après sevrage traduisant un recyclage de l'urée vers le rumen (Kouamé et al., 1984) n'apparaît dans cet essai que tardivement $\left(P_{4}\right)$ et seulement pour le lot $S$.

Les fermentations dans le rumen des veaux en cours de sevrage se développent très rapidement (2-3 semaines) après le début d'ingestion d'aliments secs, alors que les capacités d'absorption et de métabolisme de la paroi ruminale ne sont pas maximales. L'aliment concentré contenant la source de protéines la moins dégradable (tourteau de soja) conduit à des teneurs en $\mathrm{N}-\mathrm{NH}_{3}$ ruminal probablement limitantes pour la protéosynthèse microbienne.

Kouamé K. G., Patureau-Mirand P., Troccon J. L., Prugnaud J., Journet M., Pion R., 1984. Ann. Zootech., 33, 21-41.

Williams A. P., Smith R. H., 1976. Br. J. Nutr., 36, 199-209. 